BMJ Open

Diabetes

Research

\& Care

\title{
Metabolic recovery after weight loss surgery is reflected in serum microRNAs
}

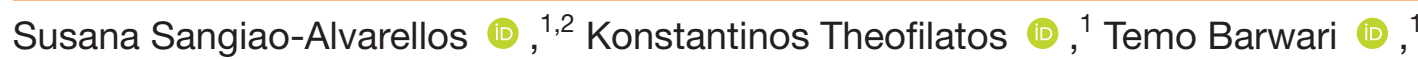
Clemens Gutmann (1), ${ }^{1}$ Kaloyan Takov (1) , ${ }^{1}$ Bhawana Singh, ${ }^{1}$ Paula Juiz-Valiña, ${ }^{2,3}$ Bárbara María Varela-Rodríguez (1) ${ }^{2,3}$ Elena Outeiriño-Blanco, ${ }^{4}$ Elisa Duregotti, ${ }^{1}$ Anna Zampetaki, ${ }^{1}$ Lukas Lunger, ${ }^{5}$ Christoph Ebenbichler (1),${ }^{5}$ Herbert Tilg, María Jesús García-Brao, ${ }^{6}$ Peter Willeit, ${ }^{7,8}$ Enrique Mena, ${ }^{6}$ Stefan Kiechl, ${ }^{8}$ Fernando Cordido, ${ }^{2,3}$ Manuel Mayr (i) ${ }^{1}$

To cite: Sangiao-Alvarellos S, Theofilatos K, Barwari T, et al. Metabolic recovery after weight loss surgery is reflected in serum microRNAs. BMJ Open Diab Res Care 2020;8:e001441. doi:10.1136/ bmjdrc-2020-001441

- Supplemental material is published online only. To view please visit the journal online (http://dx.doi.org/10.1136/ bmjdrc-2020-001441).

Received 4 April 2020 Revised 11 June 2020 Accepted 16 June 2020
Check for updates

(C) Author(s) (or their employer(s)) 2020. Re-use permitted under CC BY. Published by BMJ.

For numbered affiliations see end of article.

Correspondence to Dr Manuel Mayr; manuel.mayr@kcl.ac.uk and Dr Susana Sangiao-Alvarellos; susana.sangiao@udc.gal

\section{ABSTRACT}

Introduction Bariatric surgery offers the most effective treatment for obesity, ameliorating or even reverting associated metabolic disorders, such as type 2 diabetes. We sought to determine the effects of bariatric surgery on circulating microRNAs (miRNAs) that have been implicated in the metabolic cross talk between the liver and adipose tissue.

Research design and methods We measured 30 miRNAs in 155 morbidly obese patients and 47 controls and defined associations between miRNAs and metabolic parameters. Patients were followed up for 12 months after bariatric surgery. Key findings were replicated in a separate cohort of bariatric surgery patients with up to 18 months of follow-up.

Results Higher circulating levels of liver-related miRNAs, such as miR-122, miR-885-5p or miR-192 were observed in morbidly obese patients. The levels of these miRNAs were positively correlated with body mass index, percentage fat mass, blood glucose levels and liver transaminases. Elevated levels of circulating liver-derived miRNAs were reversed to levels of nonobese controls within 3 months after bariatric surgery. In contrast, putative adipose tissue-derived miRNAs remained unchanged (miR-99b) or increased (miR-221, miR-222) after bariatric surgery, suggesting a minor contribution of white adipose tissue to circulating miRNA levels. Circulating levels of liver-derived miRNAs normalized along with the endocrine and metabolic recovery of bariatric surgery, independent of the fat percentage reduction.

Conclusions Since liver miRNAs play a crucial role in the regulation of hepatic biochemical processes, future studies are warranted to assess whether they may serve as determinants or mediators of metabolic risk in morbidly obese patients.

\section{INTRODUCTION}

Obesity is a chronic disease and widely recognized as the largest and fastest growing public health problem in the developed and developing world. The etiology of obesity is multifactorial but unhealthy human behavior, an obesogenic environment and

\section{Significance of this study}

What is already known about this subject?

- Lipid and glucose homeostasis are controlled by a cross talk between the liver and adipose tissue. The molecular mechanisms of this cross talk remain unknown.

- Bariatric surgery is an effective treatment for obesity. It is currently unknown how it affects the liver-adipose tissue cross talk and the levels of microRNAs (miRNAs) in the circulation.

What are the new findings?

- Morbidly obese patients have elevated levels of circulating liver-derived but not adipose tissue-derived miRNAs. Liver-derived miRNAs levels positively correlate with body mass index, fat mass percentage and glucose levels.

- Bariatric surgery in obese patients restores the liverderived miRNAs in serum to healthy levels.

- Circulating levels of adipose tissue-derived miRNAs are not reduced after bariatric surgery, despite a significant weight loss. This refutes the hypothesis for a major contribution of adipose tissue to the circulating miRNA pool.

How might these results change the focus of research or clinical practice?

- Further studies on the liver-adipose tissue cross talk are warranted with a focus on liver-derived miRNAs.

- Liver-derived miRNAs may be explored as biomarkers for or drivers of metabolic syndrome in the setting of obesity.

inadequate healthcare advice are major contributors to the unprecedented increase in global overweight and obesity levels. ${ }^{1}$ Obesity contributes to the onset of metabolic disorders such as type 2 diabetes mellitus and non-alcoholic fatty liver disease (NAFLD). ${ }^{2}$ Although epidemiological correlations between obesity and insulin resistance in type 2 diabetes, NAFLD and 
metabolic syndrome are established, the cellular and molecular mechanisms that link obesity with metabolic disease remain less well explored. ${ }^{23}$

MicroRNAs (miRNAs, miR) are small non-coding RNAs that regulate gene expression at the posttranscriptional level. ${ }^{4}$ Circulating miRNAs are packaged in high-density lipoproteins, ${ }^{5}$ in protein complexes with argonaute $2^{6}$ and other RNA-binding proteins ${ }^{7}$ as well as in extracellular vesicles (EVs), including exosomes. ${ }^{8}$ Eukaryotic cells shed intact microvesicles from the cellular membrane and exosomes via the endosomal pathway. ${ }^{9}$ The composition and content of EVs vary depending on the parent cell from which they originate, as well as the cellular processes triggering their formation. It is proposed that exosomal miRNAs may be delivered to the recipient cells and participate in intercellular communication. ${ }^{1011}$

There is a well-established cross talk between adipose tissue and the liver in terms of both lipid and glucose homeostasis. Recently, Thomou et al suggested that the principal source of exosomal miRNAs in the circulation is the adipose tissue and that adipose-derived exosomal miRNAs can regulate hepatic gene expression. ${ }^{12}$ Furthermore, Zhao et al demonstrated that the levels of several EV miRNAs in the plasma of patients with NAFLD are positively correlated with body mass index (BMI), and these miRNAs enhance adipocyte lipid accumulation. ${ }^{13}$

So far, bariatric surgery offers the most effective and durable treatment for obesity ameliorating or even resolving comorbidities such as type 2 diabetes. ${ }^{3}{ }^{14} 15$ It has been demonstrated in several randomized controlled trials that bariatric surgery achieves better glycaemic control and a higher rate of type 2 diabetes remission than the exclusive use of medication. ${ }^{16}$ Some studies have investigated the effects of obesity and bariatric surgery on the pattern of both circulating and adipose tissue miRNAs. ${ }^{17-21}$ However, the beneficial potential of bariatric surgery on circulating miRNAs remains unclear. The first miRNA which was found to be related to metabolic control was miR-122. It constitutes $70 \%$ of the total miRNA content in the liver, plays a central role in lipid metabolism and was reported to be secreted in exosomes. ${ }^{22} 23$ Our previous findings demonstrated a strong association of circulating liver-derived miR-122 levels with the development of metabolic syndrome and type 2 diabetes. ${ }^{24}$ This association remained significant after multivariable adjustment, including BMI, waist-hip ratio and insulin resistance. ${ }^{24}$ It has been suggested that elevated levels of circulating miR-122 are reduced with weight loss and can be a marker of a harmful metabolic status. $^{25}$

The objective of this study was to compare circulating levels of miRNAs in morbidly obese patients and healthy volunteers. Moreover, we analyzed miRNA levels in obese patients after undergoing bariatric surgery to establish its effects on circulating miRNAs.

\section{RESEARCH DESIGN AND METHODS}

\section{Patients and sample collection}

Serum samples were collected from healthy, non-obese patients (controls), and morbidly obese patients before and after they had undergone bariatric surgery, either Roux-en-Y gastric bypass (RYGB) or sleeve gastrectomy. For the bariatric surgery group in the discovery cohort from A Coruña (Spain), serum samples were taken on the day of bariatric surgery $(n=155, T=0)$ and at various intervals after surgery. The follow-up time point at which the measurements were taken varied for different patients and was 3 months $(n=29, T=3), 6$ months $(n=27$, $\mathrm{T}=6)$ or 12 months $(\mathrm{n}=27, \mathrm{~T}=12)$ after bariatric surgery. For the validation cohort from Innsbruck (North Tyrol, Austria), stored serial serum samples were available from bariatric surgery patients with a BMI $>40 \mathrm{~kg} / \mathrm{m}^{2}$ before surgery $(n=33, T=0)$ and 12 months $(n=14, T=12)$ or 18 months $(n=19, T=18)$ after surgery, respectively. Consistent with the discovery cohort, the validation cohort also had different follow-up time points for different patients.

\section{Quantitative real-time PCR}

Total RNA from murine serum $(100 \mu \mathrm{L})$ was isolated with the miRNeasy Serum/Plasma Kit (Qiagen) according to the manufacturer's instructions. Reverse transcription was then performed with the miRCURY LNA RT kit (Exiqon, catalog no: 339340) according to the manufacturer's protocol. Real-time PCR was performed using the miRCURY Ready-to-Use PCR, Human panel I+IIV1.M platform (Exiqon) on a ViiA 7 Real-Time PCR System with 384-well block. Relative miRNA expression was determined by the comparative cycle threshold $(\Delta \Delta \mathrm{Cq})$ method by using the exogenous Caenorhabditis elegans spike-in control (cel-miR-39) for $\Delta \mathrm{Cq}$ and a calibrator consisting of an RNA pool of all samples for $\Delta \Delta \mathrm{Cq}$.

Total RNA from patient serum $(100 \mu \mathrm{L})$ was isolated with the miRNeasy Serum/Plasma Kit (Qiagen) according to the manufacturer's instructions. RNA quality was determined by spectrophotometry in an ND-1000 NANODROP 385 spectrophotometer (Thermo-Scientific). Three microliters of the extracted RNA was reverse transcribed using Megaplex RT Primers, Human Pool Set v3.0 and TaqMan MicroRNA Reverse Transcription Kit (Applied Biosystems). Prior to quantitation by real-time PCR, $1 \mu \mathrm{L}$ of undiluted RT product was preamplified using Megaplex PreAmp Primers along with the TaqMan PreAmp Master Mix (Applied Biosystems). After dilution of the sample, real-time PCR was performed using TaqMan Universal PCR Master Mix, No AmpErase UNG and specific primers to each miRNA on a ViiA 7 Real-Time PCR System with 384-well block. Relative miRNA expression was determined by the comparative $\Delta \Delta \mathrm{Cq}$ method by normalizing either to the $C$. elegans spike-in control (cel-miR-39) or to a global Cq average of 30 miRNAs: let7b, miR-122-5p, miR-125a-5p, miR-130a-3p, miR-140-5p, miR-143-3p, miR-145-5p, miR-148a-3p, miR-148b-3p, miR-150-5p, miR-191-5p miR-192-5p, miR-19b-3p, miR200a-3p, miR-20b-5p, miR-21-5p, miR-210-3p, miR-221-3p, 
miR-222-3p, miR-223-3p, miR-26a-5p, miR-27b-3p, miR29a-3p, miR-30b-5p, miR-324-5p, miR-486-5p miR-375-3p, miR-574-3p, miR-885-5p and miR-99b-5p.

\section{Lep $^{\mathrm{ob}}$ mice}

All procedures were performed in accordance with the Guidance on the Operation of the Animals (Scientific Procedures) Act 1986 (UK) with ethical approval obtained from the local ethics committee (PPL: 70/8944 to Professor Qingbo Xu). To determine whether miRNA profiles identified in bariatric patients also apply to hyperglycemic animals, we quantified miRNA levels in serum of obese mice. For this purpose, male $\mathrm{Lep}^{\mathrm{ob}}$ mice aged $8-12$ weeks $(n=15$, previously known as $o b / o b)$ were purchased from Jackson Laboratories. Male C57BL/6J mice $(n=15)$ were used as a control. Mice were kept on chow diet. A total of $1 \mathrm{~mL}$ of blood was harvested from each mouse at 14-15 weeks of age, and serum was isolated following clotting and centrifugation at $1200 \mathrm{~g}$ for $20 \mathrm{~min}$ at $4^{\circ} \mathrm{C}$. Serum samples were aliquoted and stored at $-80^{\circ} \mathrm{C}$. All experiments were performed according to protocols approved by the Institutional Committee for Use and Care of Laboratory Animals.

\section{Separation of EVs from murine serum}

A differential centrifugation-based protocol was used to separate small EVs (sEVs), large EVs (lEVs) and supernatant from mouse serum (wild type vs Lep ${ }^{\mathrm{ob}}$ mice on C57BL/6J background, all mice aged 14-15 weeks, male and on normal diet). ${ }^{26} 100 \mu \mathrm{L}$ of serum were diluted to $500 \mu \mathrm{L}$ with phosphate-buffered saline (PBS) and centrifuged at $10000 \mathrm{~g}$ for $30 \mathrm{~min}$ at $4^{\circ} \mathrm{C}$. The pellet was resuspended in $100 \mu \mathrm{L}$ PBS (=lEVs), while the supernatant was diluted to $1 \mathrm{~mL}$ with PBS and ultracentrifuged at $100000 \mathrm{~g}$ for $60 \mathrm{~min}$ at $4^{\circ} \mathrm{C}$ (Beckman Coulter Optima Max ultracentrifuge with TLA-55 rotor (k-Factor 89.5) and $9.5 \times 38 \mathrm{~mm}$ Beckman Coulter Microcentrifuge Polypropylene Tubes). The supernatant was collected, and the pellet was resuspended in $1 \mathrm{~mL}$ PBS. Ultracentrifugation was then repeated using the same settings, and the supernatant was collected and added to the supernatant obtained from the previous step to a total volume of $2 \mathrm{~mL}$ (EV-depleted supernatant). The pellet was resuspended in $100 \mu \mathrm{L}$ PBS (=sEVs). As expected, dot blot analysis confirmed that syntenin-1, a marker of sEVs, was enriched in the sEV isolates, while apolipoprotein $\mathrm{B}(\mathrm{ApoB})$, the integral membrane protein of APOB-containing lipoproteins, was depleted of sEV and IEV samples in comparison to the EV-depleted fraction (online supplemental figure 1).

\section{Data analysis}

Relative quantification values of miRNAs and clinical variables for both discovery and validation cohorts were first filtered based on a missing value criterion. Specifically, miRNAs and clinical variables with more than $50 \%$ missing values were removed from the dataset and not considered for further analysis. For example, for $\mathrm{T}=3$
C reactive protein with $76 \%$ missing values, and ApoA1 and $A$ poB with $83 \%$ missing values were not considered for further analysis. The miRNAs and clinical variables with less than $50 \%$ missing values were imputed using the KNN Impute algorithm. ${ }^{27}$ The imputation methodology was run separately for the different groups of the study. For example, for $\mathrm{T}=0 \mathrm{ApoA} 1$ with $21 \%$ missing values was imputed. The limma package ${ }^{28}$ was used to compare between different phenotypes using the empirical Bayes algorithm ${ }^{29}$ and correcting for selected covariates. Paired analysis, ie, before and after surgery, was undertaken with correction for statin use to factor the change in medications of some patients after surgery. For control (healthy) and obese patients (ie before surgery, $\mathrm{T}=0$ ), unpaired analysis was conducted. Unpaired analyses between healthy and obese participants were corrected for age, sex and statins. In all comparisons, the initial $\mathrm{P}$ values were adjusted for multiple testing using Benjamini-Hochberg $\operatorname{method}^{30}$ and a threshold of 0.05 was used for the adjusted $P$ values to infer statistically significant changes. Spearman correlation was used for studying correlation patterns and continuous variables. Point-biserial correlation $^{31}$ was used for studying correlation patterns between miRNAs and categorical variables (such as sex and type 2 diabetes). Linear regression analysis was performed to associate proteins with miR-122, and to relate miRNA changes after surgery with alterations in glucose, weight and liver transaminases, considering age, sex and statins as covariates and scaling values to zero means and SD of 1. All figures were generated with $\mathrm{R}$ programming environment and GraphPad software.

\section{RESULTS}

\section{Differences in circulating miRNAs between obese patients}

\section{and controls}

We analyzed serum samples from morbidly obese patients before bariatric surgery $(n=155)$ and from healthy participants with normal BMI as controls $(n=47)$. The clinical characteristics of all participants of this discovery cohort are shown in online supplemental table 1. A panel of 30 circulating miRNAs previously associated with the liver or adipose tissue as well as unrelated blood cellderived miRNAs were selected for analysis by quantitative PCR. The most pronounced rise in morbidly obese patients was observed for miR-122, miR-885-5p, miR-148a and miR-210 $\left(\log _{2}\right.$ fold change $(\mathrm{FC})>1.5$ and $\left.\mathrm{p}<0.001\right)$. Levels of miR-150 were markedly decreased in obese patients compared with control individuals with normal BMI (figure 1A). The key findings based on standardized miRNA concentrations to the global $\mathrm{Cq}$ average were replicated using an exogenous spike-in miRNA, cel-miR39 , for normalization (online supplemental figure 2). While miR-122 and liver-related miRNAs were among the most altered miRNAs, putative adipose tissue-derived miRNAs, ${ }^{12} 3233$ that is, miR-99b, miR-221 and miR-222, were detected at high levels in healthy human subcutaneous adipose tissue (data not shown) but were not 
A

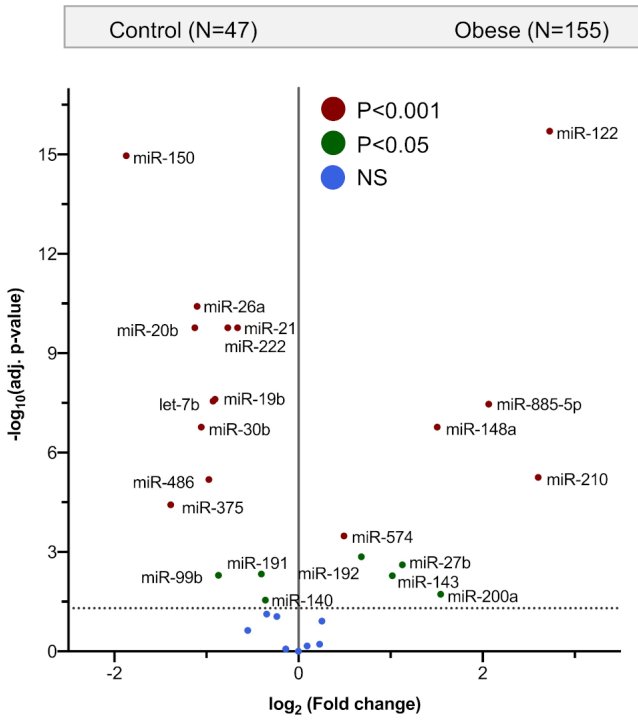

B

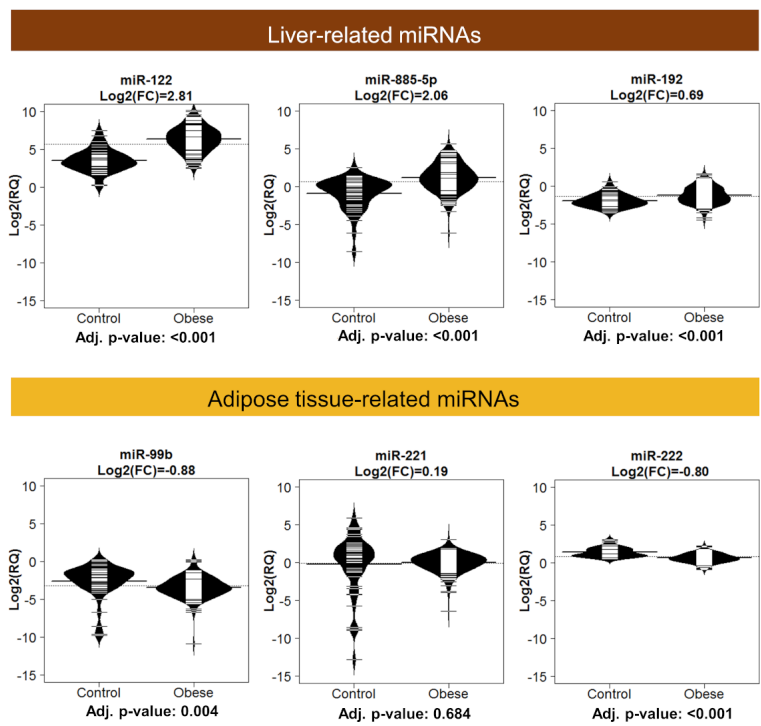

Figure 1 (A) Serum microRNAs (miRNAs) in obese patients and healthy controls. Volcano plot for differences in serum miRNAs between control $(n=47)$ and obese patients $(n=155)$ with standardized miRNA concentrations to the global Cq average. Statistical comparison was conducted using the empirical Bayes method of the limma package. P-values were corrected for multiple testing and are shown in blue, green, maroon for the different $p$-value ranges. Liver-specific miR-122 was among the miRNAs with the most elevated serum levels in obese patients compared with controls. (B) Bean plots for miRNAs normalized to the global $\mathrm{Cq}$ average. Bean plots showing elevated serum levels of liver-related (brown color) miR-122, miR-885-5p and miR-192 in obesity. The serum levels of putative adipose tissue-related miRNAs (yellow color), such as miR-99b, miR-221 and miR-222, were not elevated in obese patients. FC, fold change; NS, not significant.

elevated in serum of morbidly obese patients (figure 1B). Using cel-miR-39 as an alternative normalisation strategy, miR-221 showed an increase $\left(\log _{2}(\mathrm{FC})=1.3, \mathrm{p}=0.02\right)$, but it was relatively small in comparison to the liver-specific miR-122 $\left(\log _{2} \quad(\mathrm{FC})=3.8, \quad \mathrm{p}<0.001\right)$ and liver-related miR-885-5p $\quad\left(\log _{2} \quad(\mathrm{FC})=3.1, \mathrm{p}<0.001\right) \quad$ (online supplemental figure 3 , online supplemental table 2 ).

Correlation analysis revealed distinct clusters with positive co-expression (figure 2A). The cluster with the most pronounced obesity-induced changes included liver-specific miR-122. Based on previous studies, as well as the human miRNA transcriptomic atlas, this cluster contained other abundant liver miRNAs, that is, miR-192 and miR-885-5p. ${ }^{34}{ }^{35}$ In a correlation analysis between baseline miRNAs levels with clinical variables in obese patients (figure 2B), miRNAs correlating with miR-122, such as miR-210, miR-192 and miR-885-5p, but not miR-375, which is expressed in islet cells of the pancreas, ${ }^{36}$ were positively correlated with weight, BMI, percentage fat mass and glucose levels, and especially with liver transaminases, further supporting a relationship between these serum miRNAs and liver dysfunction.

\section{Circulating miRNA changes after bariatric surgery}

Follow-up samples were available in a subset of patients from the discovery cohort at 3 months $(n=29, T=3)$, 6 months $(n=27, T=6)$ and 12 months $(n=27, T=12)$ after bariatric surgery. Bariatric surgery reduced the elevated serum levels of miR-122, miR-192, miR-885-5p and miR-210 (figure 3; online supplemental tables $3-5)$. In contrast, many blood cell-derived miRNAs, that is, miR-21, ${ }^{37}$ and putative adipose tissue-derived miRNAs $^{123233}$ were either unchanged, that is, miR-99b, or even increased, that is, miR-221 and miR-222, after bariatric surgery (figure 3; online supplemental table 6). Again, miRNA concentrations that were standardized to the global $\mathrm{Cq}$ average (online supplemental table 6) were in agreement with results that used cel-miR-39 as an alternative normalization strategy (online supplemental table 7). Given a nearly $40 \%$ reduction in the average BMI after 12 months (from $49.6 \pm 0.62$ before to $30.9 \pm 0.91 \mathrm{~kg} / \mathrm{m}^{2}$ after bariatric surgery), our results suggest that adipose tissue may not be the main source of these circulating miRNAs.

To confirm our key findings, we performed further miRNAs measurements in an independent validation cohort of obese patients with samples before bariatric surgery $(n=33)$ and at follow-up after 12 months $(n=14)$ or 18 months $(n=19)$, respectively. Their clinical characteristics are shown in online supplemental table 8. A comparison of the results from the discovery and the validation cohort is presented in online supplemental table 9 , confirming a significant reduction of circulating liver-derived miRNAs after bariatric surgery (online supplemental tables 10 and 11). In contrast, analysis of the putative adipose tissue-derived miRNAs showed no significant changes for miR-99b, miR-222 and miR-221 at either time point despite a pronounced and sustained weight loss over the 18 months of follow-up after bariatric surgery. 

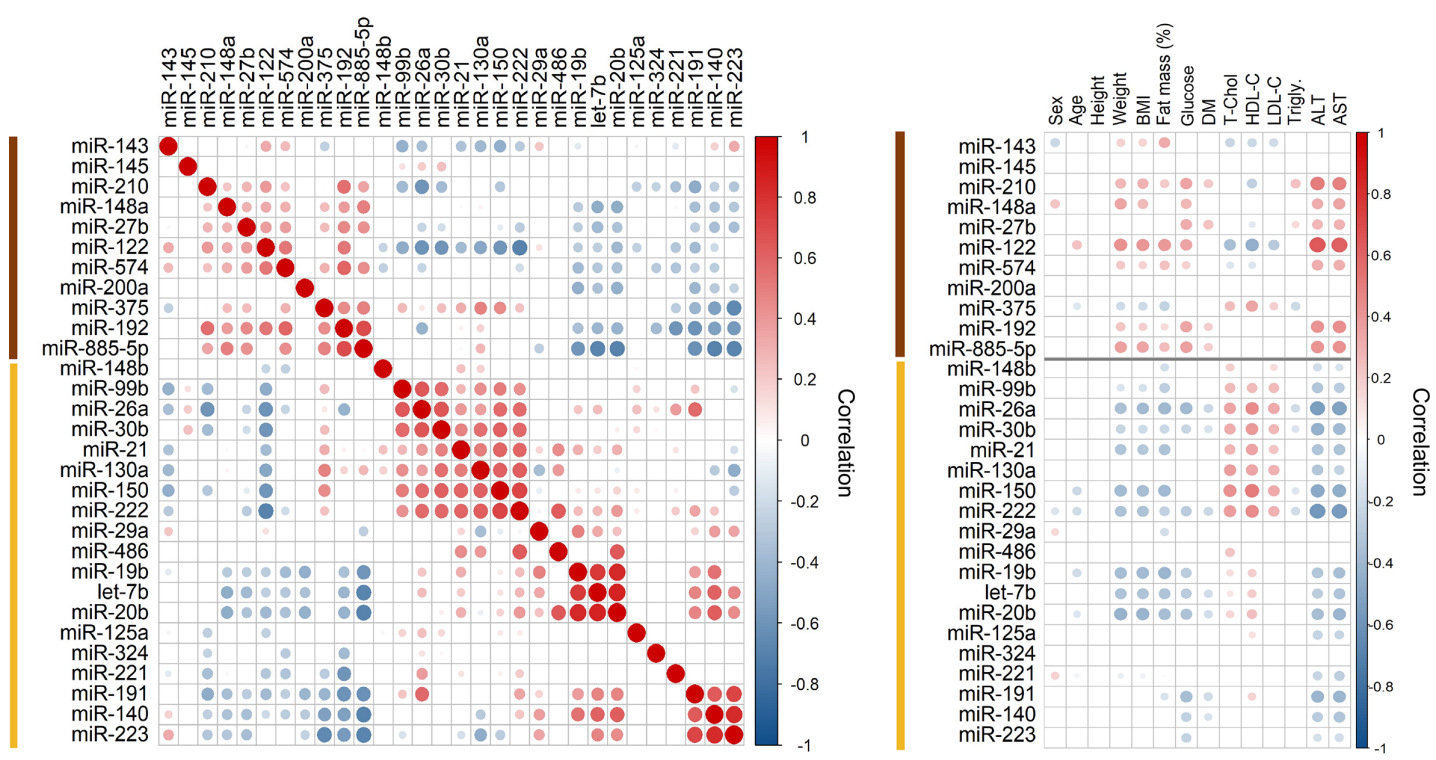

Figure 2 (A) Pairwise Spearman correlation of serum microRNA (miRNA) levels. Hierarchical clustering analysis and heat map matrix illustrating positive and negative co-expression and intracluster and intercluster relationships of pairwise comparisons of miRNAs before bariatric surgery. Only significant correlations are shown ( $p<0.05$, normalized to the global Cq average). The sizes of the circles highlight the strength of correlation. Two distinct clusters emerge: one smaller cluster (brown color) is composed of liver-specific miR-122 and other liver-related miRNAs such as miR-885-5p. The larger cluster (yellow color) contains a wide range of miRNAs from different cellular origins, including the putative adipose tissue-related miRNAs miR99b, miR-221 and miR-222. (B) Correlations of serum miRNAs to clinical variables. Spearman correlation plot showing only significant correlations before bariatric surgery $(p<0.05$, normalized to the global Cq average). For the categorical variables sex and diabetes, point-biserial correlation was used. The cluster of liver-related miRNAs (highlighted in brown) is positively correlated (red color) with weight, BMI, percentage fat mass, blood glucose and liver transaminases. The different sizes of the circles highlight the strength of correlation. There was no positive association between BMl and percentage fat mass with serum levels of putative adipose tissue-related miRNAs, miR-99b, miR-221 and miR-222. ALT, alanine aminotransferase; AST, aspartate aminotransferase; BMI, body mass index; DM, type 2 diabetes mellitus; fat mass (\%), percentage fat mass; HDL-C, high-density lipoprotein cholesterol; LDL-C, low-density lipoprotein cholesterol; T-Chol, total cholesterol.

\section{Compartmentalization of circulating miRNAs}

Baseline levels of putative adipose tissue-derived miRNAs were lower in obese patients compared with non-obese controls, that is, miR-99b and miR-222, or were not different, that is, miR-221. Moreover, circulating levels of miR-99b did not change after bariatric surgery despite the substantial weight loss, while miR-221 and miR-222 even increased after bariatric surgery. A possible explanation for these results is that these serum miRNAs are either not adipose tissue-derived or adipose tissuederived exosomes are not a major contributor to the total circulating miRNA content as suggested previously. ${ }^{12}$ To address the latter question, we harvested serum from 14 to 15 week old wild type and obese-hyperglycemic Lep ${ }^{\mathrm{ob}}$ mice and separated EVs by differential centrifugation. The profound increase of liver miRNAs observed in morbidly obese patients could independently be replicated in serum samples of obese-hyperglycemic Lep ${ }^{\text {ob }}$ mice (figure 4A). Next, we conducted miRNA analysis in sEVs and lEVs as well as in the EV-depleted supernatant. Liver-derived miR-192-5p and miR-122-5p were predominantly detected in the EV-depleted supernatant. MiR-885-5p was not included as this miRNA is not present in mice. For the putative adipose tissue-derived
miRNAs, miR-221, miR-99b and miR-222, their relative miRNA content in EVs was higher than liver-derived miRNAs (figure 4B), but there was no significant change in sEVs isolated from serum of Lep ${ }^{\mathrm{ob}}$ mice compared with wild-type controls (online supplemental figure 4). In fact, a reduction of miR-221 and miR-222 was observed in lEVs from Lep ${ }^{\mathrm{ob}}$ mice, in opposing direction to the corresponding serum levels. Thus, EVs are a minor contributor to the circulating levels of these putative adipose tissue-derived miRNAs.

\section{DISCUSSION}

The circulating miRNAs that showed the most profound changes in morbidly obese patients formed a distinct co-expression cluster that included liver-specific miR-122, which correlated with body weight, BMI, percentage fat mass and liver transaminase levels. The observed drop of liver miRNAs within the first 3 months after bariatric surgery indicated a metabolic recovery following a timeline correlating with the normalization of transaminase levels. At 3 months, the patients achieved on average a $20 \%$ reduction in their BMI. Additional weight loss thereafter had no further effect on circulating levels of liver 


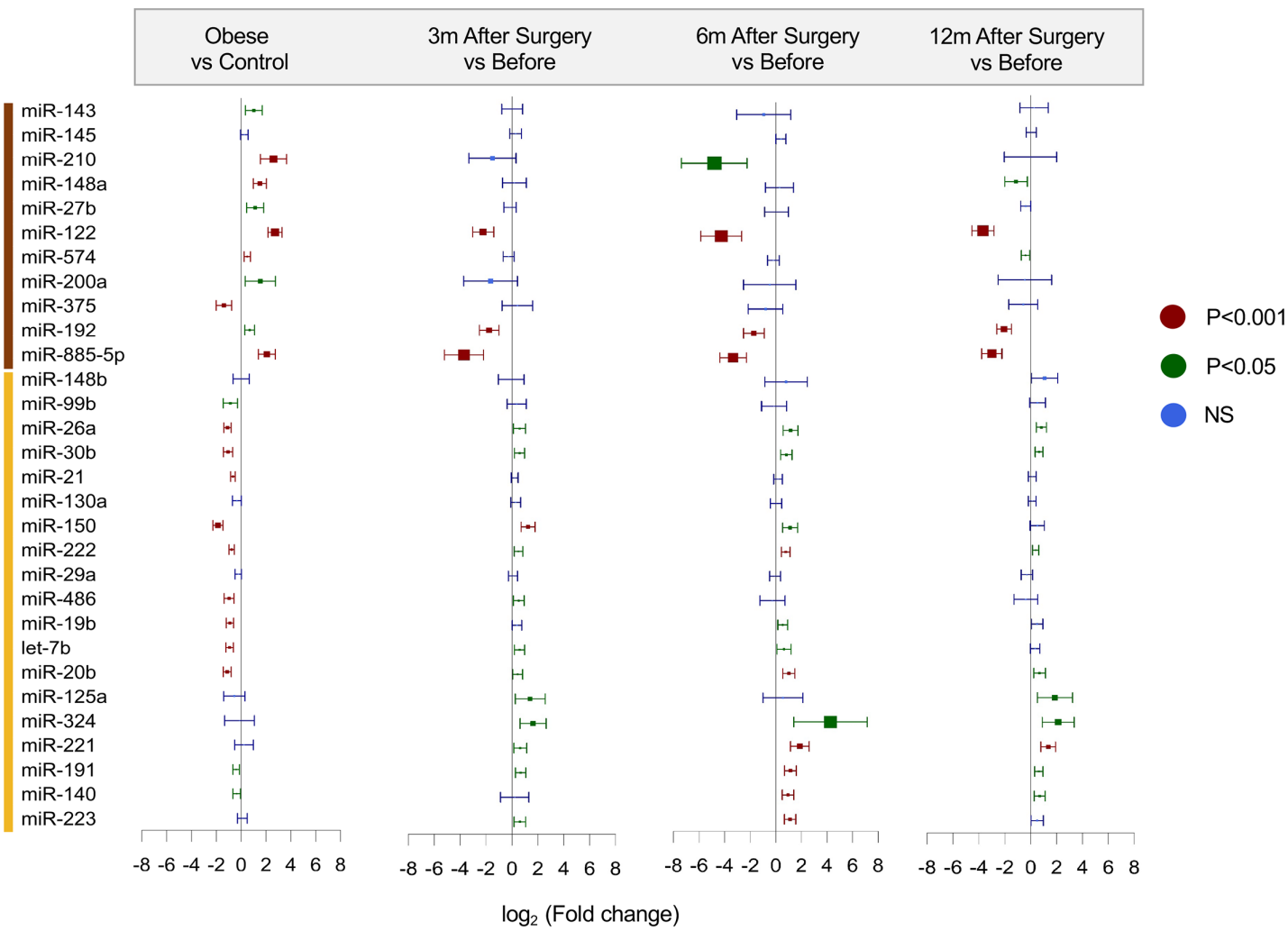

$\log _{2}$ (Fold change)

Figure 3 Effect of obesity and bariatric surgery on serum microRNA (miRNA) levels. Forest plots with the corresponding relative abundance ratios ( $\log _{2}$-fold change, normalized to the global Cq average). Levels of miR-122, miR-885-5p and miR192 fell within the first 3 months after bariatric surgery. These miRNAs were part of the cluster containing liver-related miRNAs (highlighted in brown color). At 3 months, patients achieved a reduction in body mass index (BMI) of approximately $20 \%$. In contrast, miRNAs within the cluster containing the putative adipose tissue-related miRNAs (yellow color) showed no reduction even at 12 months of follow-up despite a reduction in BMI of almost $40 \%$. The p-values are corrected for multiple testing and the box sizes (shown in blue, green, maroon for the different $p$-value ranges) are relative to the absolute $\log _{2}$-fold change. NS, not significant.

miRNAs. In contrast, candidate adipose-derived miRNAs, such as miR-99b, which Thomou et al ${ }^{12}$ suggested to be a previously undescribed form of adipokine, did not decline after bariatric surgery despite the dramatic weight loss after a 12-18-month follow-up.

Previous work reported higher miR-122, miR-148a and miR-192 circulating levels in obesity. ${ }^{38}$ Altered blood miR-122 and miR-885-5p levels have also been implicated in a variety of liver diseases, including drug-induced liver injury and NAFLD. ${ }^{39} 40$ Prevalence of NAFLD increases in parallel with obesity, metabolic syndrome and diabetes. ${ }^{41}{ }^{42}$ In patients with NAFLD, miR-122 and miR-192 were among the most upregulated miRNAs in serum. ${ }^{43}$ Elevated miR-122 and miR-192 levels were also part of a plasma signature that predicted postoperative liver dysfunction. ${ }^{44}$ Obese patients showed reduced levels of miR-122 1 year after RYGB while before surgery miR-122 circulating levels correlated with aspartate aminotransferase and alanine aminotransferase. ${ }^{45}$ Other circulating miRNAs that showed profound changes in our study have also been reported to increase with liver damage, that is, miR-210, miR-148a, miR-143, miR-574, miR-27b and miR-200a. ${ }^{39} 40434446$
Thomou $e a^{12}$ suggested a predominant adipose tissue origin of circulating exosomal miRNAs based on the use of adipose tissue-specific Dicer knockout (AdicerKO) mice. Dicer is required for the conversion of most, but not all pre-miRNAs to mature miRNAs. ${ }^{47}$ Liver-related miRNAs with profound changes after bariatric surgery, that is, miR148a, miR-122, miR-210, were also decreased in exosomes from AdicerKO mice. In the study by Thomou et $a l,{ }^{12}$ exosomal miRNA profiling in patient serum returned only one miRNA, which was also found in our study to be significantly increased in obesity and reduced after bariatric surgery: miR-210 was upregulated in patients with congenital generalized lipodystrophy but not dysregulated in HIV-associated lipodystrophy $(\mathrm{n}=4$ each and $\mathrm{n}=4$ controls). To the best of our knowledge, the putative adipose tissue-derived miRNAs have not been measured in morbidly obese patients so far. Despite the fact that we detected high levels of adipose tissue-derived miRNAs in healthy human subcutaneous adipose tissue, the present data in morbidly obese patients refute that serum miRNAs are predominantly adipose tissue derived or that white adipose tissue-derived exosomes are a major contributor to the total circulating levels of miRNAs. 
A

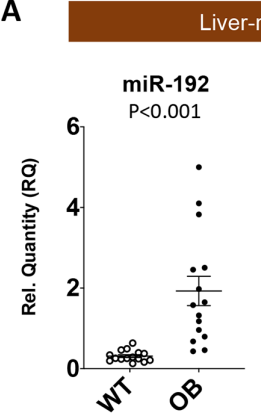

Liver-related miRNAs

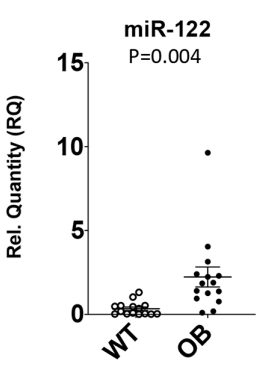

B

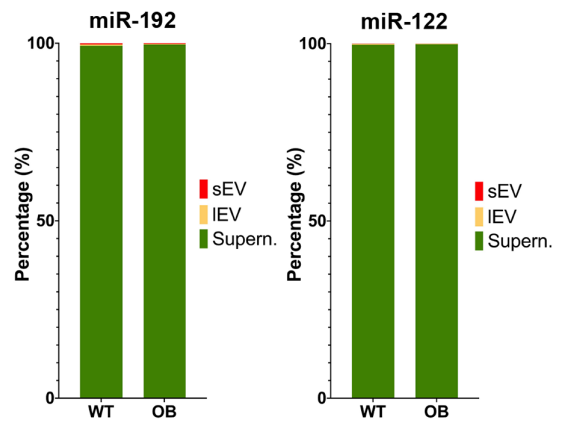

Adipose tissue-related miRNAs

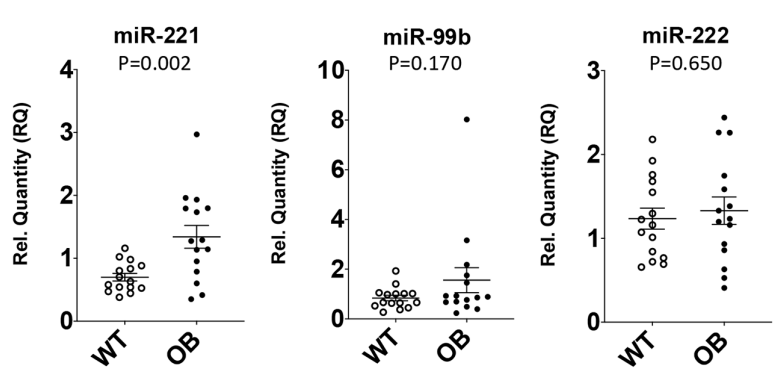

Figure 4 (A) Differences in serum microRNAs (miRNAs) between control C57BL/6 (WT, wild type) and Lep ${ }^{\mathrm{ob}}$ mice (OB, obese). (B) Compartmentalization of serum miRNAs. Differential centrifugation was used to separate small extracellular vesicles (sEVs), large extracellular vesicles (IEVs) and EV-depleted supernatant (Supern.) from mouse serum (WT vs Lep ${ }^{\mathrm{ob}}$ mice, all male mice aged 14-15 weeks fed on chow diet).

One potential explanation for the discrepancy between our findings in morbidly obese patients and the ones reported by Thomou $e t a l^{2}$ in AdicerKO mice is that brown adipose tissue is prominent in mice. Rodents and other small mammals have copious brown fat, whereas humans have very little after childhood. ${ }^{48}$ Thus, while liver miRNAs remain conserved across species, the composition of adipose-derived miRNAs, would be different between mice and humans. Given the analytical challenges and the lack of scalable methods to obtain pure vesicular preparations, we did not attempt to isolate EVs from human serum. However, the putative adipose tissue-derived miRNAs miR-99b, miR-221 and miR-222 showed no increase in the vesicle fractions of hyperglycemic Lep $^{\text {ob }}$ mice. It should be noted that, although plasma concentrations of putative adipose-tissue derived miRNAs did not change with weight loss, changes in adipose tissue function could still affect circulating miRNAs. Dynamic changes in the production or removal of circulating miRNAs might not be reflected in static concentrations.

In summary, morbid obesity was associated with a profound increase in liver-derived miRNAs. The elevated serum levels of liver-derived miRNAs dropped within 3 months after bariatric surgery. This was associated with a recovery of parameters related to liver dysfunction and reversal of type 2 diabetes, but not to further weight loss, suggesting that the main cause is a metabolic improvement rather than the loss of white adipose tissue.
Author affiliations

${ }^{1}$ King's British Heart Foundation Centre, School of Cardiovascular Medicine and Sciences, King's College London, London, UK

${ }^{2}$ Endocrine, Nutritional and Metabolic Diseases Group, Department of

Physiotherapy, Medicine and Biomedical Sciences, Faculty of Health Sciences, University of A Coruña, A Coruña, Spain

${ }^{3}$ Instituto de Investigación Biomédica de A Coruña (INIBIC), A Coruña, Spain

${ }^{4}$ Department of Endocrinology, A Coruña University Hospital, A Coruña, Spain

${ }^{5}$ Department for Internal Medicine I, Medizinische Universität Innsbruck, Innsbruck, Austria

${ }^{6}$ Department of Digestive and General Surgery, A Coruña University Hospital, A Coruña, Spain

${ }^{7}$ Department of Public Health and Primary Care, University of Cambridge, Cambridge, UK

${ }^{8}$ Department of Neurology, Medical University of Innsbruck, Innsbruck, Austria

Contributors SS-A, TB, CG, KTa, AZ and PJ-V researched data. KTh, BS, SK, CE and PW analyzed data. BMV-R, EO-B, ED, LL, HT, MJG-B and EM reviewed and edited the manuscript. SS-A, SK, FC and MM wrote the manuscript. MM is the guarantor of this work and, as such, had full access to all the data in the study and takes responsibility for the integrity of the data and the accuracy of the data analysis.

Funding SS-A was recipient of a travel/visiting scientist fellowship "José Castillejo" from the Ministerio de Educación, Cultura y Deporte-Spain. Project № Pl16/00884 was awarded to FC and SS-A integrated in the Spanish National Plan for Scientific Research, Development and Technological Innovation 2013-2016 and funded by the ISCIII. CG is funded by a British Heart Foundation (BHF) PhD studentship (FS/18/60/34181). MM is a BHF Chair Holder (CH/16/3/32406) with BHF program grant support (RG/16/14/32397). MM was awarded a BHF Special Project grant to participate in the ERA-CVD Transnational Grant "MacroERA: Noncoding RNAs in cardiac macrophages and their role in heart failure" and is part of the Marie Skłodowska-Curie Innovative Training Network TRAIN-HEART (http://train-heart.euhttp://train-heart.eu) as well as a networks on "MicroRNAbased Therapeutic Strategies in Vascular Disease" and on "Defining the Roles of Smooth Muscle Cells and other Extracellular Matrix Producing Cells in Late Stage Atherosclerotic Plaque Formation" funded by the Fondation Leducq. This work was supported by the National Institute of Health Research Biomedical Research 
Centre based at Guy's and St Thomas' NHS (National Health Service) Foundation Trust and King's College London in partnership with King's College Hospital and an R\&D K-Centre and the excellence initiative VASCage (Centre for Promoting Vascular Health in the Ageing Community, project number 868624) of the Austrian Research Promotion Agency FFG (COMET program-Competence Centers for Excellent Technologies) funded by the Austrian Ministry for Transport, Innovation and Technology, the Austrian Ministry for Digital and Economic Affairs and the federal states Tyrol (via Standortagentur), Salzburg and Vienna (via Vienna Business Agency).

Competing interests PW, SK and MM are named inventors on a licensed patent held by Medical University Innsbruck and King's College London for the use of miR122 as a biomarker of metabolic risk (EP2430453B1, US8546089, EP15193448.6) MM filed and licensed patent applications on miRNAs as biomarkers (EP2776580 B1, DE112013006129T5, GB2524692A, EP2576826 B, JP2013-513740). All other authors have no disclosures.

Patient consent for publication Not required.

Ethics approval The study was conducted in accordance with the Declaration of Helsinki and was approved by the Institutional Ethics Committees (Galicia, reference 2014/135; Innsbruck reference AN163 197/4.11 (3350a)). All patients gave written informed consent. All procedures were performed in accordance with the Guidance on the Operation of the Animals (Scientific Procedures) Act 1986 (UK) with ethical approval obtained from the local ethics committee (PPL: 70/8944 to Professor Qingbo Xu).

Provenance and peer review Not commissioned; externally peer reviewed.

Data availability statement Data are available on reasonable request. Deidentified participant data with corresponding microRNA measurements are available from the author MM (ORCID identifier 0000-0002-0597-829X).

Open access This is an open access article distributed in accordance with the Creative Commons Attribution 4.0 Unported (CC BY 4.0) license, which permits others to copy, redistribute, remix, transform and build upon this work for any purpose, provided the original work is properly cited, a link to the licence is given, and indication of whether changes were made. See: https://creativecommons.org/ licenses/by/4.0/.

\section{ORCID iDs}

Susana Sangiao-Alvarellos http://orcid.org/0000-0003-1207-4667

Konstantinos Theofilatos http://orcid.org/0000-0001-6799-0553

Temo Barwari http://orcid.org/0000-0003-2279-8677

Clemens Gutmann http://orcid.org/0000-0003-0675-8632

Kaloyan Takov http://orcid.org/0000-0002-8642-6306

Bárbara María Varela-Rodríguez http://orcid.org/0000-0001-5350-4534

Christoph Ebenbichler http://orcid.org/0000-0001-5025-7929

Manuel Mayr http://orcid.org/0000-0002-0597-829X

\section{REFERENCES}

1 Bray GA, Heisel WE, Afshin A, et al. The science of obesity management: an Endocrine Society scientific statement. Endocr Rev 2018;39:79-132.

2 Heymsfield SB, Wadden TA. Mechanisms, pathophysiology, and management of obesity. N Engl J Med 2017;376:254-66.

3 Reges O, Greenland P, Dicker D, et al. Association of bariatric surgery using laparoscopic banding, Roux-en-Y gastric bypass, or laparoscopic sleeve gastrectomy vs usual care obesity management with all-cause mortality. JAMA 2018:319:279-90.

4 Bartel DP. MicroRNAs: genomics, biogenesis, mechanism, and function. Cell 2004:116:281-97.

5 Vickers KC, Palmisano BT, Shoucri BM, et al. MicroRNAs are transported in plasma and delivered to recipient cells by highdensity lipoproteins. Nat Cell Biol 2011;13:423-33.

6 Arroyo JD, Chevillet JR, Kroh EM, et al. Argonaute2 complexes carry a population of circulating microRNAs independent of vesicles in human plasma. Proc Natl Acad Sci U S A 2011;108:5003-8.

7 Wang K, Zhang S, Weber J, et al. Export of microRNAs and microRNA-protective protein by mammalian cells. Nucleic Acids Res 2010;38:7248-59

8 Mittelbrunn M, Gutiérrez-Vázquez C, Villarroya-Beltri C, et al. Unidirectional transfer of microRNA-loaded exosomes from $T$ cells to antigen-presenting cells. Nat Commun 2011;2:282

9 Mathieu M, Martin-Jaular L, Lavieu G, et al. Specificities of secretion and uptake of exosomes and other extracellular vesicles for cell-tocell communication. Nat Cell Biol 2019;21:9-17.
10 Bayraktar R, Van Roosbroeck K, Calin GA. Cell-to-cell communication: microRNAs as hormones. Mol Oncol 2017;11:1673-86.

11 Pegtel DM, Cosmopoulos K, Thorley-Lawson DA, et al. Functional delivery of viral miRNAs via exosomes. Proc Natl Acad Sci U S A 2010;107:6328-33.

12 Thomou T, Mori MA, Dreyfuss JM, et al. Adipose-derived circulating miRNAs regulate gene expression in other tissues. Nature 2017;542:450-5.

13 Zhao Y, Zhao M-F, Jiang S, et al. Liver governs adipose remodelling via extracellular vesicles in response to lipid overload. Nat Commun 2020;11:719.

14 Schauer PR, Bhatt DL, Kirwan JP, et al. Bariatric surgery versus intensive medical therapy for diabetes - 5-year outcomes. $N$ Engl $J$ Med 2017;376:641-51.

15 Adams TD, Davidson LE, Hunt SC. Weight and metabolic outcomes 12 years after gastric bypass. N Engl J Med 2018;378:93-6.

16 Sudlow A, le Roux CW, Pournaras DJ. Review of multimodal treatment for type 2 diabetes: combining metabolic surgery and pharmacotherapy. Ther Adv Endocrinol Metab 2019;10:204201881987540.

17 Bae $\mathrm{Y}-\mathrm{U}, \mathrm{Kim} \mathrm{Y}$, Lee $\mathrm{H}$, et al. Bariatric surgery alters microRNA content of circulating exosomes in patients with obesity. Obesity 2019;27:264-71.

18 Langi G, Szczerbinski L, Kretowski A. Meta-analysis of differential miRNA expression after bariatric surgery. J Clin Med 2019;8:1220.

19 Atkin SL, Ramachandran V, Yousri NA, et al. Changes in blood microRNA expression and early metabolic responsiveness 21 days following bariatric surgery. Front Endocrinol 2018;9:773.

20 Hubal MJ, Nadler EP, Ferrante SC, et al. Circulating adipocytederived exosomal microRNAs associated with decreased insulin resistance after gastric bypass. Obesity 2017;25:102-10.

21 Alkandari A, Ashrafian H, Sathyapalan T, et al. Improved physiology and metabolic flux after Roux-en-Y gastric bypass is associated with temporal changes in the circulating microRNAome: a longitudinal study in humans. BMC Obes 2018;5:20.

22 Gallo A, Tandon M, Alevizos I, et al. The majority of microRNAs detectable in serum and saliva is concentrated in exosomes. PLOS One 2012;7:e30679.

23 Huang X, Yuan T, Tschannen M, et al. Characterization of human plasma-derived exosomal RNAs by deep sequencing. BMC Genomics 2013;14:319.

24 Willeit P, Skroblin P, Moschen AR, et al. Circulating microRNA-122 is associated with the risk of new-onset metabolic syndrome and type 2 diabetes. Diabetes 2017;66:347-57.

25 Hess AL, Larsen LH, Udesen PB, et al. Levels of circulating miR-122 are associated with weight loss and metabolic syndrome. Obesity 2020;28:493-501.

26 Théry C, Amigorena S, Raposo G, et al. Isolation and characterization of exosomes from cell culture supernatants and biological fluids. Curr Protoc Cell Biol 2006; Chapter 3:3.22.1-3.22.29

27 Andridge RR, Little RJA. A review of hot Deck imputation for survey non-response. Int Stat Rev 2010;78:40-64.

28 Smyth GKet al. Limma: linear models for microarray data. In: Gentleman R, Carey VJ, Wolfgang $\mathrm{H}$, et al, eds. Bioinformatics and computational biology solutions using $R$ and Bioconductor. New York: Springer, 2005: 397-420.

29 Carlin BP, Louis TA. Bayes and empirical Bayes methods for data analysis. Chapman and Hall/CRC, 2010.

30 Ferreira JA, Zwinderman AH. On the Benjamini-Hochberg method. Ann Stat 2006;34:1827-49.

31 Kornbrot D. Point Biserial Correlation. In: Encyclopedia of statistics in behavioral science. John Wiley \& Sons L, 2005.

32 Meerson A, Traurig M, Ossowski V, et al. Human adipose microRNA-221 is upregulated in obesity and affects fat metabolism downstream of leptin and TNF- $\alpha$. Diabetologia 2013;56:1971-9.

33 Ortega FJ, Moreno-Navarrete JM, Pardo G, et al. MiRNA expression profile of human subcutaneous adipose and during adipocyte differentiation. PLoS One 2010:5:e9022.

34 Lizio M, Abugessaisa I, Noguchi S, et al. Update of the FANTOM web resource: expansion to provide additional transcriptome atlases. Nucleic Acids Res 2019;47:D752-8.

35 Schueller F, Roy S, Vucur M, et al. The role of miRNAs in the pathophysiology of liver diseases and toxicity. Int J Mol Sci 2018;19. doi:10.3390/ijms19010261. [Epub ahead of print: 16 Jan 2018].

36 Poy MN, Eliasson L, Krutzfeldt J, et al. A pancreatic islet-specific microRNA regulates insulin secretion. Nature 2004;432:226-30.

37 Barwari T, Eminaga S, Mayr U, et al. Inhibition of profibrotic microRNA-21 affects platelets and their releasate. $\mathrm{JCl}$ Insight 2018;3. 
38 Ortiz-Dosal A, Rodil-García P, Salazar-Olivo LA. Circulating microRNAs in human obesity: a systematic review. Biomarkers 2019;24:499-509.

39 do Amaral AE, Cisilotto J, Creczynski-Pasa TB, et al. Circulating miRNAs in nontumoral liver diseases. Pharmacol Res 2018;128:274-87.

40 Loosen SH, Schueller F, Trautwein C, et al. Role of circulating microRNAs in liver diseases. World J Hepatol 2017;9:586-94.

41 Brunt EM, Wong VW-S, Nobili V, et al. Nonalcoholic fatty liver disease. Nat Rev Dis Primers 2015;1:15080.

42 Rinella ME. Nonalcoholic fatty liver disease: a systematic review. JAMA 2015;313:2263-73.

43 Pirola CJ, Fernández Gianotti T, Castaño GO, et al. Circulating microRNA signature in non-alcoholic fatty liver disease: from serum non-coding RNAs to liver histology and disease pathogenesis. Gut 2015;64:800-12.
44 Starlinger P, Hackl H, Pereyra D, et al. Predicting postoperative liver dysfunction based on blood-derived microRNA signatures. Hepatology 2019;69:2636-51.

45 Rega-Kaun G, Kaun C, Jaegersberger G, et al. Roux-en-Y-bariatric surgery reduces markers of metabolic syndrome in morbidly obese patients. Obes Surg 2020;30:391-400.

46 Raitoharju E, Seppälä I, Lyytikäinen L-P, et al. Blood hsa-miR122-5p and hsa-miR-885-5p levels associate with fatty liver and related lipoprotein metabolism-The Young Finns Study. Sci Rep 2016;6:38262

47 Kim Y-K, Kim B, Kim VN. Re-Evaluation of the roles of Drosha, export in 5, and Dicer in microRNA biogenesis. Proc Natl Acad Sci U S A 2016;113:E1881-9.

48 Zhang F, Hao G, Shao M, et al. An adipose tissue atlas: an imageguided identification of human-like bat and beige depots in rodents. Cell Metab 2018;27:252-62. 